\title{
Multifractal analysis of skin temperature fluctuations of women breasts with and without tumor
}

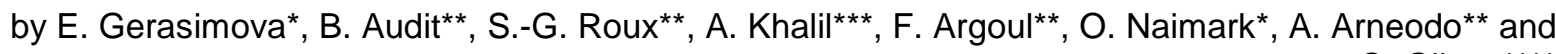 \\ O. Gileva $a^{* * *}$ \\ * Institute of continuous media mechanics UB RAS, 1 Acad. Korolev Street, 614013, Perm, Russia, \\ egerasimova@icmm.ru \\ ** Université de Lyon, F-69000 Lyon, France and Laboratoire de Physique, ENS de Lyon, CNRS, UMR 5672, \\ Allée d'Italie F-69007 Lyon, France, alain.arneodo@ens-lyon.fr \\ *** Department of Mathematics and Statistics, University of Maine, Orono, ME 04469, USA, akhalil@jax.org \\ **** Perm state medical academy, 39 Kuibyshev Street, 614990, Perm, Russia, ogileva@rambler.ru
}

\begin{abstract}
The wavelet transform modulus maxima method was used to reveal changes in skin temperature dynamics of woman breasts with and without malignant tumor. The statistics of temperature temporal fluctuations about the cardiogenic and vasomotor perfusion oscillations do not change across time-scales for cancerous breasts as the signature of homogeneous monofractal fluctuations. This contrasts with the continuous change of temperature fluctuation statistics observed for healthy breasts as the hallmark of complex multifractal scaling. The study shows that multifractal analysis of dynamic IR imaging can be effectively used for preliminary screening in asymptomatic women to identify those with risk of breast cancer.
\end{abstract}

\section{Introduction}

Breast cancer is the most common type of cancer among women and, despite recent advances in the medical field, it remains one of the deadliest of all cancers due to the inherent limitations of currently used screening techniques. The radiological interpretation of X-ray mammograms often leads to over-diagnosis and, as a consequence, to unnecessary traumatic and painful biopsies. Based on the results of the study a computer-aided multifractal analysis of dynamic infrared (IR) imaging can be recommended for identification of women with risk of breast cancer. Application of a wavelet-based multi-scale method allows to analyze the temporal fluctuations of breast skin temperature collected from patients with breast cancers and some healthy volunteers. The multifractal complexity of temperature fluctuations observed in healthy breasts is lost in breasts with malignant tumor that can be used for diagnostic purposes as diagnostic value. In addition to potential clinical impact, these results shed a new light on physiological changes that may occur before the morphological alterations in breast cancer genesis.

\section{Methods of analysis}

\subsection{The wavelet transform modulus maxima method (1D)}

The wavelet transform (WT) is a mathematical microscope, which owing to its ability to filter out low-frequency trends in the analyzed signal $\Sigma$, is well suited for the analysis of complex non-stationary time-series such as those found in physiological systems. The wavelet transform (WT) is used to perform a space-scale analysis by expanding a signal in terms of wavelets that are constructed from the general analyzing wavelet $\psi$ by means of dilations and translations. The WT of a function $\Sigma$ is defined as:

$$
W_{\psi}\left(t_{0}, a\right)=\frac{1}{a} \int_{-\infty}^{+\infty} \Sigma(t) \psi\left(\frac{t-t_{0}}{a}\right) d t
$$

where $x_{0}$ and $a(>0)$ are the space and scale parameters, respectively. Here, we use the compactly supported version of the Mexican hat, which has two vanishing moments.

The WTMM method [1,2] consists in investigating the scaling behavior of some partition function defined in terms of wavelet coefficients:

$$
Z(q, a)=\sum_{l \in L(a)}|W(t, a)|^{q} \sim a^{\tau(q)},
$$

where $q \in \mathbb{R}$. The sum is taken over the WT skeleton $L(a)$ defined, at each fixed scale a, by the local maxima of $\left|W_{\psi}(t, a)\right|$. These WTMM are connected across the scales by lines, which are called maxima lines $l_{t}(a)$. Along these lines the WTMM behave as $a^{h(t)}$ in the limit $\mathrm{a} \rightarrow 0^{+}$, where $h(x)$ is the Hölder exponent characterizing the singularity of $\Sigma$ located at position $t$ to which $I_{t}($ a) points.

The Legendre transform of the scaling function $\tau(q)$ in Eq. (2), is the singularity spectrum $D(h)$ defined as the Hausdorf dimension of the set of points $x$ where the Hölder exponent value is $h$ : 
$D(h)=\min _{q}[q h-\tau(q)]$.

As an alternative strategy, one can compute the mean quantities $h(q, a)$ and $D(q, a)$ :

$$
\begin{gathered}
h(q, a)=\sum_{l \in L(a)} \ln \left|W_{\psi}(x, a)\right| \hat{W}_{\psi}(q, L, a) \sim h(q) \ln a, \\
D(q, a)=\sum_{l \in L(a)} \hat{W}_{\psi}(q, L, a) \ln \left|\hat{W}_{\psi}(q, L, a)\right| \sim D(q) \ln a,
\end{gathered}
$$

where $W_{\psi}(q, L, a)=\left|W_{\psi}(x, a)\right|^{q} / Z(q, a)$ is a Boltzmann weight coefficients computed from the wavelet transform skeleton. By analyzing the scaling behavior of these quantities, in the limit $a \rightarrow 0^{+}$, it is possible to link spectra $h(q)$ and $D(q)$ and consequently the $D(q)$ spectrum.

As commonly done for noise signals, the wavelet analysis was performed on the cumulative (or integral) $\Sigma$ of the temperature time-series using the second-order compactly supported version $\psi_{(3)}^{(2)}$ of the Mexican hat $[3,4]$. Hence the singularities with possible negative Hölder exponent $-1<h<0$, become singularities with $0<h_{c}=h+1<1$ in the cumulative.

The $\tau(q)$ data were fitted by the quadratic approximation $\tau(q)=-c_{0}+c_{1} q-c_{2} q^{2} / 2$, where the coefficients $c_{n}>0$. The corresponding singularity spectrum has a characteristic single humped shape curve $D(h)=c_{0}-\left(h-c_{1}\right)^{2} / 2 c_{2}$, where $c_{0}=-\tau(0)$ is the fractal dimension of the support of singularities of $\Sigma, c_{1}$ is the value of $h$ that maximizes $D(h)$ and $c_{2}$, the so-called intermittency coefficient, characterizes the width of the $D(h)$ spectrum, i.e. the signature of multifractal properties is $c_{2} \neq 0$.

\subsection{Monofractal and multifractal functions}

Homogeneous (mono) fractal functions, i.e. functions with singularities of the same Hölder exponent $H$ are characterized by a linear $\tau(q)$ curve $(h=\partial \tau / \partial q=H)$.A nonlinear $\tau(q)$ is the signature of nonhomogeneous functions that exhibit multifractal properties, meaning that the Hölder exponent $h(x)$ is a fluctuating quantity that depends on $x$.

Since calculating $Z$ (q, a) (Eq. (2)) amounts to computing the $q$-order moment of the wavelet transform modulus maxima probability density function $\rho_{a}\left(W_{\psi}(\cdot, a)\right)$ at scale $a$, monofractal scaling implies that the shape of this pdf does not depend on the scale $a$, formally expressed by the self-similarity relationship:

$$
a^{H} \rho_{a}\left(a^{H} W\right)=\rho(W),
$$

where $\rho(W)$ is a "universal" pdf that does not depend on a.

\section{Study design}

33 patients (from 37 to 83 years old) with histologically confirmed malignant tumor (invasive ductal and/or lobular cancer) and 14 healthy volunteers (from 23 to 79 years old; control group) were included into the study [4]. The tumors were found at different depths from $1 \mathrm{~cm}$ down to $12 \mathrm{~cm}$ with a size varying from $1.2 \mathrm{~cm}$ up to $6.5 \mathrm{~cm}$. Both (healthy and cancerous) breasts of patients with cancer and both volunteers' healthy breast were imaged with a InSb photovoltaic (PV) detector camera according our original technique. Each image set comprised 30000 image frames during the 10 min immobile imaging phase. We grouped single-pixel temperature time-series into $8 \times 8$ pixel ${ }^{2}$ squares covering the entire breast. Reported results are averaged over 64 temperature time-series in these $8 \times 8$ subareas.

\section{Results}

\subsection{Power spectrum analysis breast skin temperature dynamics}

We analyzed 1-pixel temperature time-series taken from $8 \times 8$ pixels ${ }^{2}$-squares covering patient entire breasts. As expected, these time-series generally fluctuate at a higher temperature when recorded in the tumor region of a malignant breast (Fig. 1, a) than in a symmetrically localized square on the opposite breast (Fig. 1, b) as well as on a healthy breast (Fig. 1, c). When averaging the corresponding power-spectra over the 64 pixels of the considered squares, we observed for the two breasts of patient 20 (age 56) and the healthy breast of volunteer 14 (age 60), a rather convincing $1 / f^{\beta}$ power-law scaling over a range of frequencies that extends from the characteristic human respiratory frequency $(>0.3 \mathrm{~Hz})$ up to the cross-over frequency $(<4 \sim \mathrm{Hz})$ towards (instrumental) white noise (Fig. 1, $d$ ). As confirmed by the WTMM method (Fig. 3,a), the exponent $\beta=\tau(q=2)=0.62 \pm 0.19$ found in the malignant breast is smaller than in the opposite breast of patient $20, \beta=1.32 \pm 0.11$, and in the volunteer 14 healthy breast, $\beta=1.22 \pm 0.11$. This difference looks quite significant and very promising in a discriminatory perspective. Unfortunately, the histograms of $\beta$ values obtained for all $8 \times 8$ pixels ${ }^{2}$ squares covering 33 cancerous breasts, the 32 opposite breasts (patient 6 had mastectomy of right breast) and the 28 volunteer healthy breasts are quite similar (Fig. 1,e) with mean values 

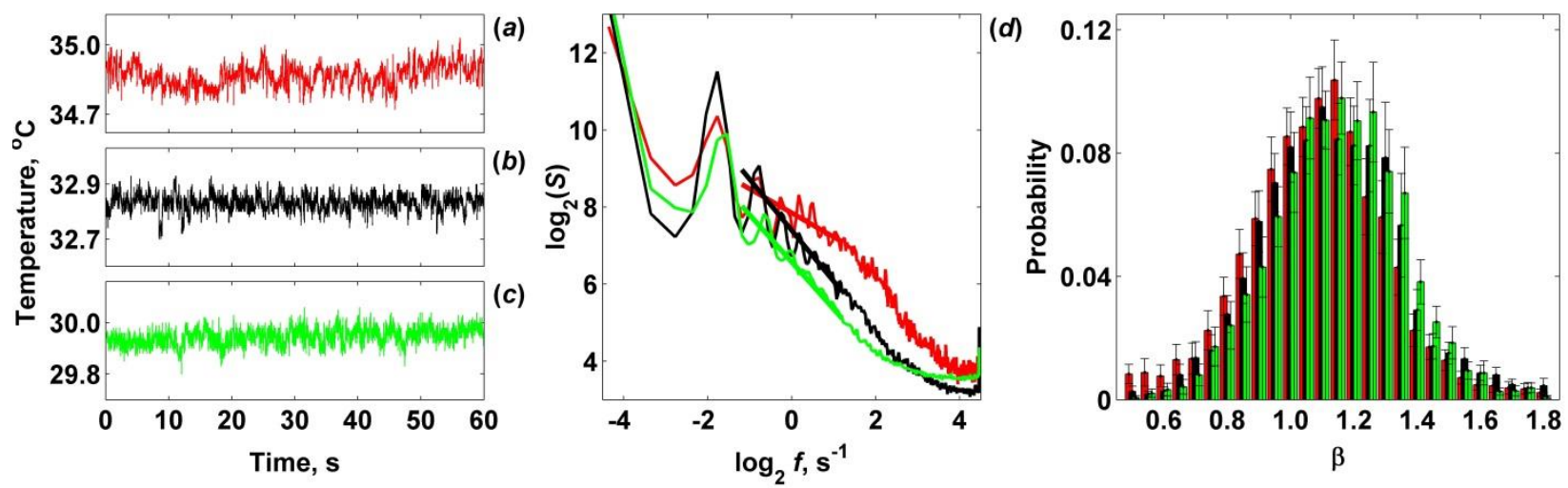

(e)

Fig. 1. Power spectrum analysis of IR temperature time-series. Comparative analysis of the cancerous right breast (red) and healthy left breast (black) of patient 20 (age 56) and of the healthy right breast (green) of volunteer 14 (age 60). (ac) 1 min portions of pixel temperature time-series. (d) Averaged temperature power spectra in a $8 \times 8$ pixels square. The straight lines correspond to power-law scaling $1 / f^{\beta}$. (e) Comparative power spectrum analysis of temperature time-series recorded on both breasts of 33 patients with breast cancer and 14 healthy volunteers. Normalized histograms of power-

law scaling exponent $\beta$ values obtained in the breast with malignant tumor (red: $N=40328 \times 8$ pixels ${ }^{2}$ squares), the opposite breasts (black: $N=3606$ ) and the healthy breasts (green: $N=3185$ ).

$\bar{\beta}=1.09 \pm 0.01$ (cancer), $1.14 \pm 0.01$ (opposite) and $1.14 \pm 0.01$. Indeed these histograms extend over a rather wide range of $\beta$ values $(0.5 \leq \beta \leq 1.9)$.

\subsection{Multifractal analysis of dynamic thermograms of breasts}

When applying the WTMM method to the cumulative of these temperature time-series, we confirmed that the partition functions $Z(q, a)$ (Eq. (2)) display nice scaling properties for $q=-1$ to 5 , over a range of time-scales that we strictly limited to $[0.43 \mathrm{~s}, 2.30 \mathrm{~s}]$ for linear regression fit estimates in a logarithmic representation (Fig. 2, a). The $\tau(q)$ soobtained are well approximated by quadratic spectra (Fig. 3, a). For the malignant breast of patient $20, \tau(q)$ is nearly linear as quantified by a very small value of the intermittency coefficient $c_{2}=(4.4 \pm 0.6) \cdot 10^{-3}$. This signature of monofractality is confirmed, when respectively plotting $h(q, a)$ and $D(q, a)$ (Eq. (4)) vs $\log _{2} a$, in Fig. 2, $b$ and $c$, where the slopes $h(q)=c_{1}=0.81 \pm 0.01$ and $D(h)=0.99 \pm 0.03$, do not significantly depend on $q$, meaning that the $D(h)$ singularity spectrum nearly reduces to a single point $D\left(h=c_{1}=0.81\right)=1$ (Fig. $\left.3, b\right)$. This monofractal diagnosis is confirmed when comparing the WT pdfs obtained at different time-scales (Fig. 4,a); according to Eq. (6), they all collapse on a single curve when using the exponent $H=c_{1}$ (Fig. 4, d).
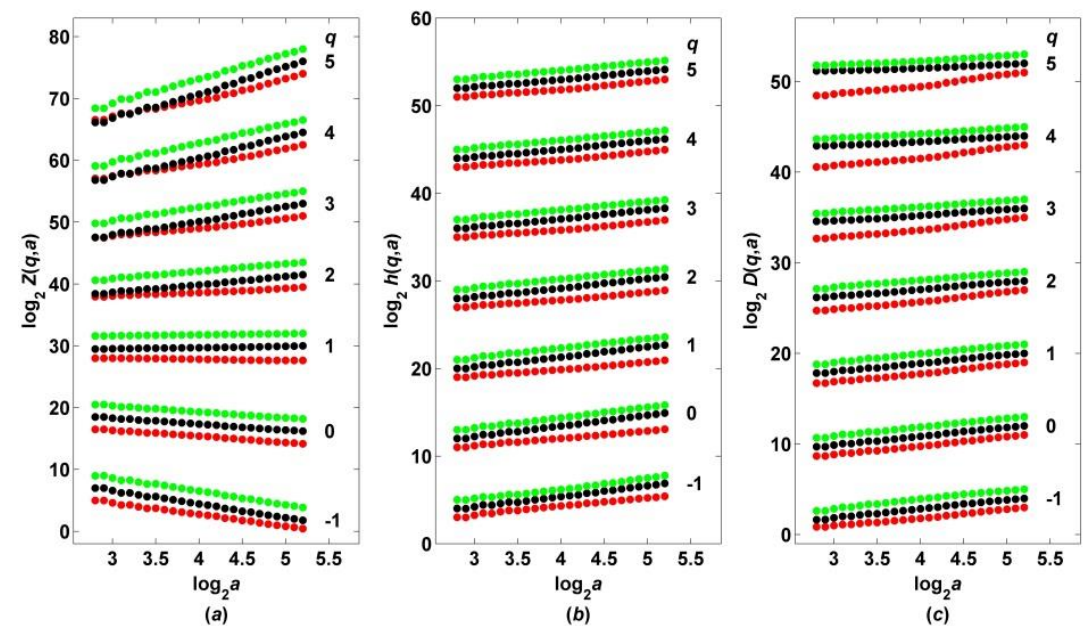

Fig. 2. Multifractal analysis of cumulative IR temperature time-series in a $8 \times 8$ pixels $^{2}$ square in malignant right breast (red) and opposite left breast (black) of patient 20 and of the healthy right breast (green) of volunteer 14. (a) $\log _{2} Z(q, a)$ vs $\log _{2}$ a (Eq. (2)). (b) $\log _{2} h(q, a)$ vs $\log _{2}$ a (Eq. (4)). (c) $\log _{2} D(q, a)$ vs $\log _{2}$ a (Eq. (5)). 


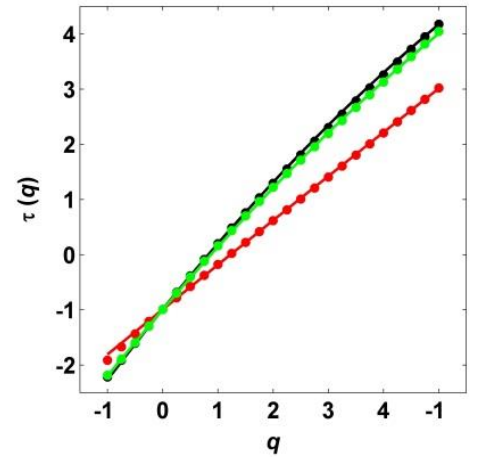

(a)

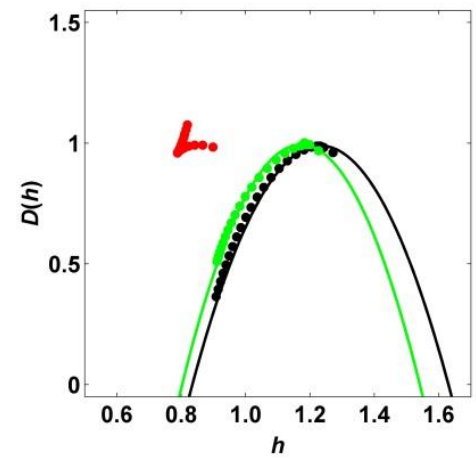

(b)

Fig. 3. Multifractal analysis of cumulative IR temperature time-series in a $8 \times 8$ pixels ${ }^{2}$ square in malignant right breast (red) and opposite left breast (black) of patient 20 and of the healthy right breast (green) of volunteer 14. (a) $\tau(q) v s q$ estimated by linear regression fit of $\log _{2} Z(q, a)$ vs $\log _{2}$ a over a range of time-scales [0.3, 3] s (Fig. 2, a). (b) $D(h)$ vs $h$

(Fig. 2, b, c).

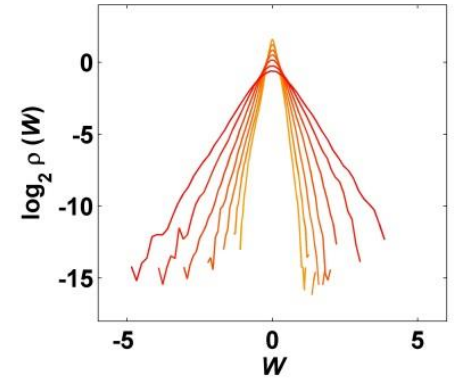

(a)

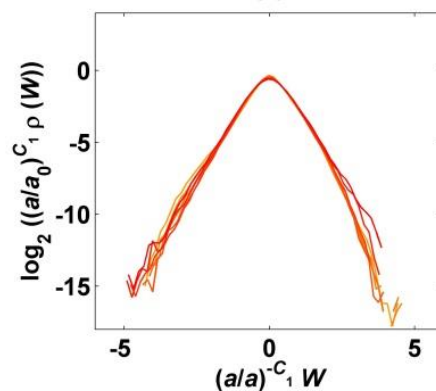

(d)

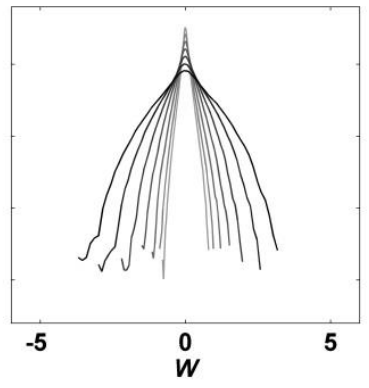

(b)

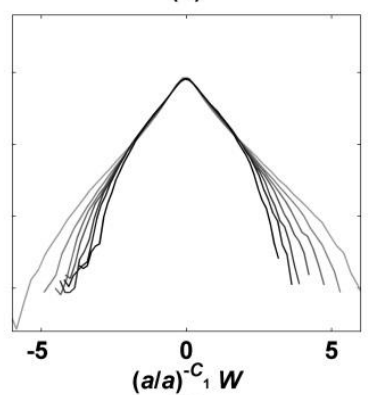

(e)

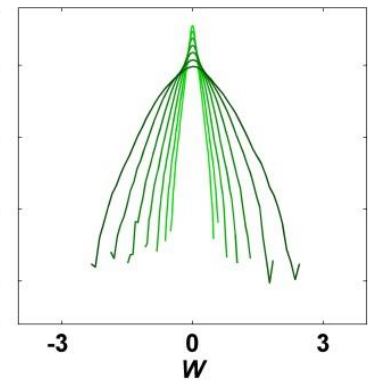

(c)

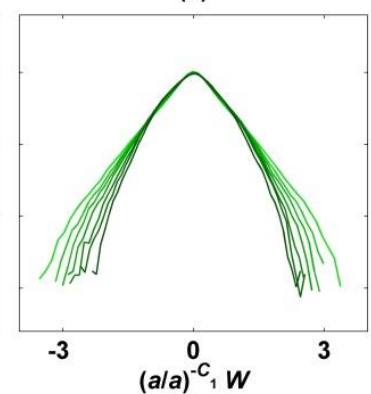

(f)

Fig. 4. Evolution of the temperature WT coefficient pdfs across time-scales. Pdfs of WT coefficients $W$ and rescaled WT coefficients $\left(W /\left(a / a_{0}\right)^{c_{1}}\right)$ of cumulative temperature of $(a, d)$ the cancerous right breast of patient $20: c_{1}=0.81,(b, e)$ the opposite left breast of patient 20: $c_{1}=1.23$, and $(c, f)$ the healthy right breast of volunteer 14: $c_{1}=1.17$. The different curves correspond to seven different scales from $a=0.43 \$ s$ to $2.30 \mathrm{~s}$, larger scales are in darker shades; $a_{0}=0.43 \mathrm{~s}$. The pdfs were averaged over a $8 \times 8$ pixels ${ }^{2}$ square.

\subsection{Comparative statistical analysis of skin temperature dynamics in women breasts with and without} malignant tumor

The results of our comparative wavelet-based multifractal analysis of (cumulative) temperature fluctuations over the 33 cancerous breasts, the 32 opposite breasts (no right breast for patient 6 ) and the 28 volunteer healthy breasts are reported in Fig. 5. In Fig. 5, a, the corresponding histograms of $c_{1}$ values extend over a rather wide range $0.6 \leq c_{1} \leq 1.8$ but turn out to be quite similar with mean values $\bar{c}_{1}=1.066 \pm 0.002$ (cancer), $1.104 \pm 0.002$ (opposite) and $1.103 \pm 0.002$ (healthy). This is reminiscent to the similar histograms that were also obtained for the power-spectrum exponent $\beta$ (Fig. 1,e). In contrast, the intermittency parameter $c_{2}$ has a definite discriminatory power. The histogram for 


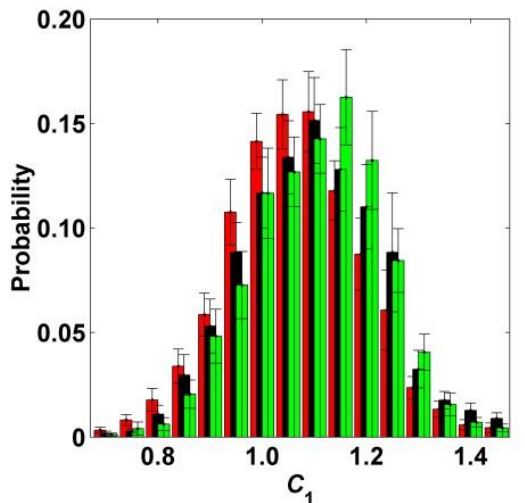

(a)

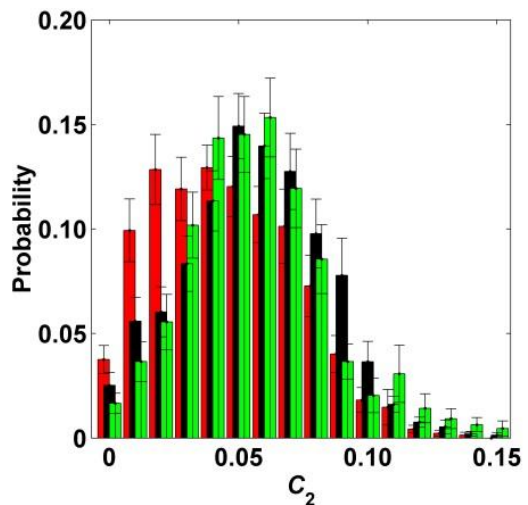

(b)

Fig. 5. Differential multifractal signature of temperature temporal fluctuations on breasts with and without tumor.

Comparative analysis of both breasts of 33 patients with breast cancer and 14 healthy volunteers. (a) Normalized histograms of $c_{1}$ values obtained in the breasts with a malignant tumor (red: $N=40328 \times 8$ pixels ${ }^{2}$ squares), the opposite breasts (black: $N=3606$ ) and the healthy breasts (green: $N=3185)$. (b) Normalized histograms of $c_{2}$ values.

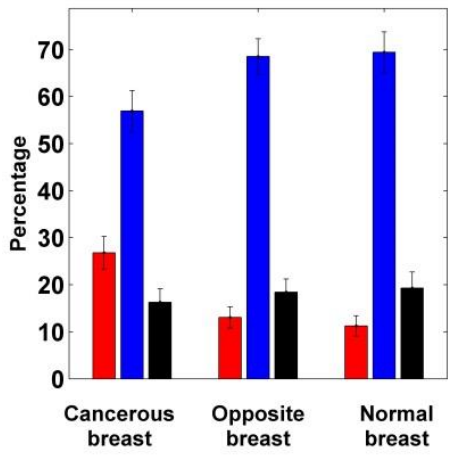

(a)

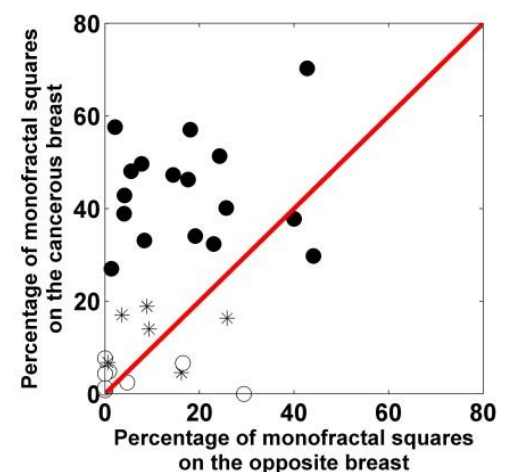

(b)

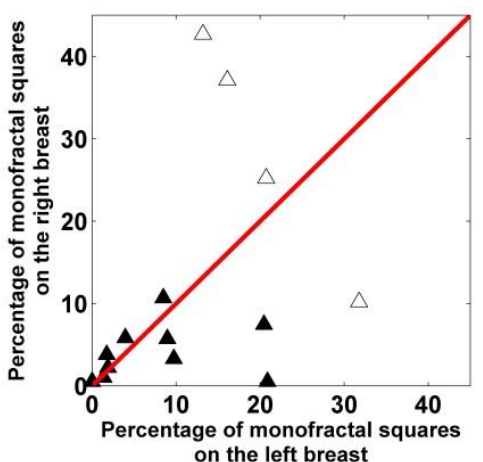

(c)

Fig. 6. (a) Percentage of "monofractal" (red: $c_{2}<0.03$ ), "multifractal" (blue: $c_{2} \geq 0.03$ ) and "no scaling" (black) $8 \times 8$ pixels ${ }^{2}$ squares in cancerous, opposite and healthy breasts. (b) Percentage of "monofractal" $8 \times 8$ pixels ${ }^{2}$ squares in the cancerous breast vs the percentage in the opposite breast of each patient; the symbols have the following meaning:

(•) 17 patients with a percentage of monofractal squares on the cancerous breast larger than the mean value $26.8 \%$; (*) 7 patients with the percentage of monofractal squares on the cancerous breast smaller than $26.8 \%$ but well localized on the tumor region; (०) 8 false negatives. (c) Percentage of "monofractal" $8 \times 8$ pixels ${ }^{2}$ squares in the right breast vs the percentage in the left breast of each healthy volunteer; the symbols have the following meaning: ( $\mathbf{\Delta}) 10$ volunteers with a percentage of monofractal squares on the cancerous breast smaller than the mean value $26.8 \% ;(\Delta) 4$ volunteers with the percentage of monofractal squares on the cancerous breast larger than $26.8 \%$.

cancerous breasts $\left(\bar{c}_{2}=0.045 \pm 0.001\right)$ is definitely shifted towards smaller values relative to the ones for opposite healthy breasts $\left(\bar{c}_{2}=0.056 \pm 0.001\right)$ and normal breasts of healthy volunteers $\left(\bar{c}_{2}=0.058 \pm 0.001\right)$ (Fig. $\left.5, b\right)$. The smallvalues of $c_{2}$ dominate in cancerous breasts compared with healthy breasts, confirming that cancerous breasts are enriched in squares where temperature fluctuations display significantly reduced multifractal properties. This justifies that we considered $c_{2}=0.03$ as the threshold below (resp. above) which a square was qualified as monofractal (resp. multifractal) (Fig. 6, a).

According proposed classification $8 \times 8$ pixel $^{2}$ squares covered breast thermograms were color coded and represented as a dots in the $\left(c_{1}, c_{2}\right)$ plane (Fig. 7). High percentage of squares $(49.7 \%)$ in the cancerous breast displays monofractal temperature fluctuations with small intermittency coefficient values $\left(c_{2}<0.03\right.$ in Fig. $\left.7, d\right)$, while only few of those squares are found in the opposite healthy breast $(7.7 \%$ in Fig. $7, e)$ and in the healthy breast of volunteer $(11 \%$ in Fig. $7, f)$. In thermograms of opposite healthy breast and volunteer's normal breast a majority of squares $(89.4 \%$ and $65 \%$ respectively) manifests multifractal scaling with intermittency coefficient $c_{2} \geq 0.03$ (Fig. $7, b$ and $c$ ). $43.1 \%$ of the squares in the cancerous breast also display multifractal temperature fluctuations as observed for healthy breasts (Fig. 7, a). Note that for each breast, a small percentage $(<20 \%)$ of (white) squares were removed from our analysis because of lack of scaling. 
Cancerous breast

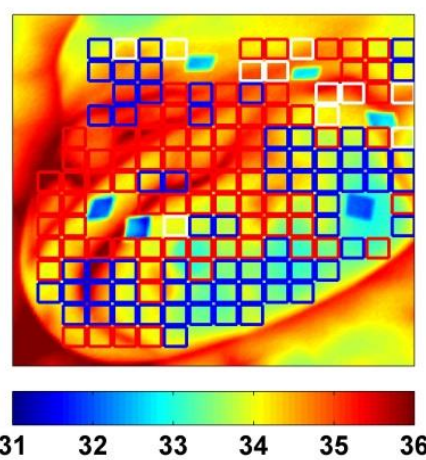

(a)

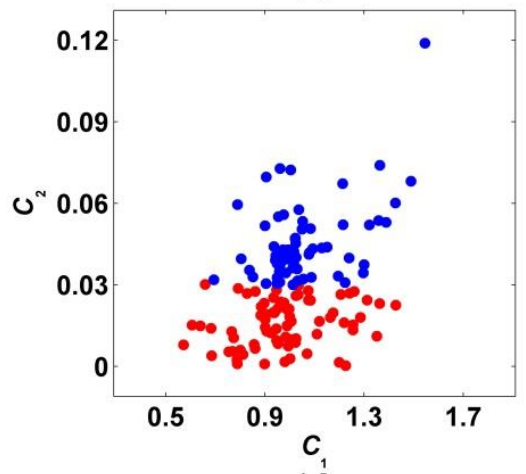

(d)
Opposite breast

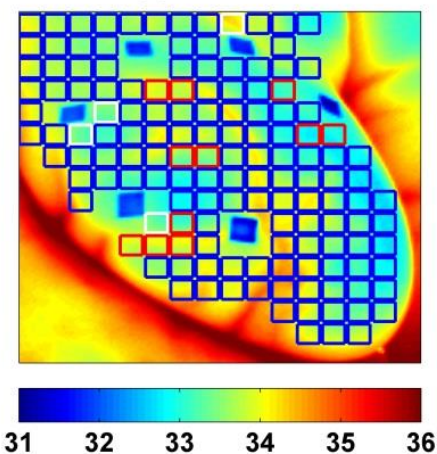

(b)

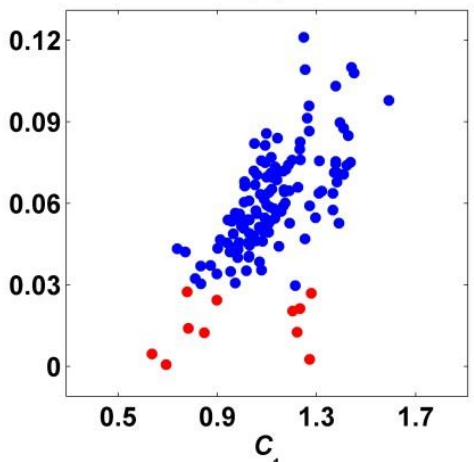

(e)
Normal breast

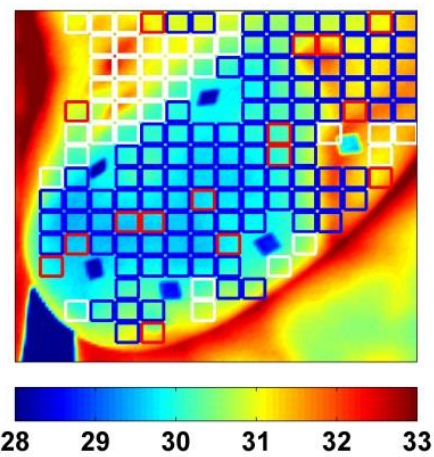

(c)

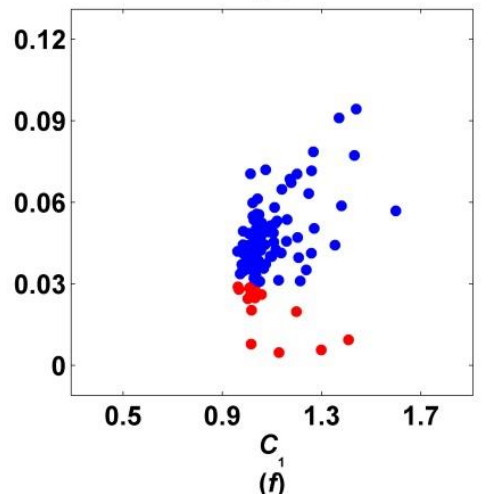

$(f)$

Fig. 7. Breast-wide multifractal analysis of skin temperature temporal fluctuations. As estimated from the $t(q)$ spectra computed with the WTMM method (Fig. 3, b), $8 \times 8$ pixels ${ }^{2}$ squares covering the entire breast are color coded (a-c) and represented as a dots in the $\left(c_{1}, c_{2}\right)$ plane $(d-f)$. The colors have the following meaning: red: $c_{2}<0.03$, blue: $c_{2} \geq 0.03$ and white: no scaling (see text). $(a, d)$ Cancerous right breast of patient 20: the tumor is located in the upper outer quadrant;

$(b, e)$ healthy left breast of patient 20; (c, f) healthy right breast of volunteer 14.

Comparative statistical analysis of monofractal and multifractal squares distribution in breast thermograms has shown, that the percentage of monofractal (red) squares covering cancerous breasts $(26.8 \pm 3.5 \%)$ is about twice higher than the ones covering opposite healthy breasts $(13.1 \pm 2.3 \%)$ and normal breasts in patients of control group $(11.3 \pm 2.2 \%)$. This excess is compensated by a smaller percentage of multifractal (blue) squares in cancerous breasts $(56.9 \pm 4.4 \%)$ than in opposite healthy breasts $(68.5 \pm 3.8 \%)$ and normal breasts $(69.4 \pm 4.3 \%)$ in volunteers of control group (Fig. 6, a).

We compared the percentages of monofractal squares in cancerous and opposite healthy breasts and found that 25 patients have higher quantity of monofractal squares in the cancerous breast (Fig. 6, b). Indeed, 18 of them are characterized with a percentage of monofractal squares greater than the mean value $(26 \%)$ were found (Fig. $6, b)$. Among the other 15 cancerous breasts, 7 have a smaller percentage of monofractal squares but well localized in the tumor region ( $*$ in Fig. $6, b$ ). The remaining 8 cancerous breasts were detected as false negatives due to small percentage of monofractal squares and their distant location with regard to tumor region ( $\circ$ in Fig. $6, b$ ). 4 of them were corresponded to rather deep tumors $(6 \mathrm{~cm}, 7 \mathrm{~cm}, 8 \mathrm{~cm}$ and $12 \mathrm{~cm}$ depth) in breasts with massive adipose tissue that can explain the absence of significant changes in skin temperature dynamics. Interestingly that in 5 of opposite healthy breasts the high (>26\%) percentage of monofractal squares was detected that can be an early sign of pathology and need following check-ups. As a control, we reproduced this comparative analysis on the two normal breasts of the 14 healthy volunteers (Fig. 6, c). Only $4(\Delta$ in Fig. $6, c)$ volunteers manifest high $(>26 \%)$ percentage of monofractal squares, while in most of them ( $\boldsymbol{\Delta}$ in Fig. $6, c)$ this value did not exceed $10 \%$. Thus, the results of breast dynamic infrared thermography interpreted in terms of multifractal analysis promote $76 \%$ sensitivity and $86 \%$ specificity in breast cancer detection.

\section{Conclusions}

The wavelet-based multifractal analysis of dynamic IR thermograms is able to differenciate cancerous breasts with monofractal skin temperature fluctuations characterized by a unique singularity exponent and healthy breasts with multifractal skin temperature fluctuations characterized by a wide range of singularity exponents. This finding correlates with the results of a similar wavelet-based multifractal analysis of human heart beat dynamics in [5], which shows that the 
multifractal character and nonlinear properties of the healthy heart rate are lost in pathological condition of congestive heart failure.

We are very grateful to Perm Regional Government for the contract №C26/614 by 19.12.2012 "Multiscale approaches in mechanobiology for early cancer diagnosis" and to Russian Foundation of Basic Research (grant 13-0196044).

\section{REFERENCES}

[1] Muzy J.-F., Bacry E., Arneodo A. "Wavelets and multifractal formalism for singular signals: Application to turbulence data". Physical Review Letters, vol. 67, pp. 3515-3518, 1991.

[2] Arneodo A., Audit B., Decoster N., Muzy J.-F., Vaillant C. "A wavelet based multifractal formalism: Application to DNA sequences, satellite images of the cloud structure and stock market data" in The Science of Disasters: Climate Disruptions, Heart Attacks, and Market Crashes edited by Bunde A., Kropp J., Schellnhuber H.J., pp. 26-102, Berlin: Springer Verlag, 2002.

[3] Gerasimova E., Audit B., Roux S., Khalil A., Argoul F., Naimark O., Arneodo A. "Multifractal analysis of dynamic infrared imaging of breast cancer". Europhysics Letters, vol. 104, pp. 68001, 2013.

[4] Gerasimova E., Audit B., Roux S., Khalil A., Gileva O., Argoul F., Naimark O., Arneodo A. "Waveletbased multifractal analysis of dynamic infrared thermograms to assist in early breast cancer diagnosis". Frontiers in Physiology (in press, 2014).

[5] Ivanov P. C., Amaral L. A., Goldberger A. L., Havlin S., Rosenblum M. G., Struzik Z. R., Stanley H. E. "Multifractality in human heartbeat dynamics". Nature, vol. 399, pp. 461-465, 1999. 